\title{
DENSITIES AND ABUNDANCES OF HOT COMETARY IONS IN THE COMA OF P/HALLEY
}

\author{
M. NEUGEBAUER, R. GoldSTERN AND B. E. GoldSTEN \\ Jet Propulsion Laboratory, California Institute of Technology \\ S. A. FUSELIER \\ Lockheed Palo Alto Research Laboratory \\ H. BALSIGER \\ University of Bern \\ AND \\ W.-H. IP \\ Max-Planck-Institut für Aeronomie
}

Submitted to The Astrophysical Journal

Received:

Accepted: $\frac{\text { Oec. }}{\text { in press } 1990}$ 
On its flight by P/Halley, the Giotto spacecraft carried a High Energy Range Spectrometer (HERS) for measuring the properties of cometary ions picked up by the solar wind in the nearly collisionless regions of the coma. Preliminary estimates of the ion densities observed by HERS have now been reevaluated and extended; density profiles along the Giotto trajectory are presented for 13 values of ion mass/charge. Comparison with the physical-chemical model of the interaction of sunlight and the solar wind with the comet by Schmidt et al. (1988) reveal that, with the exception of protons and $\mathrm{H}_{2}{ }^{+}$, all ion densities were at least an order of magnitude higher than predicted. The high ion densities cannot be explained on the basis of compression of the plasma, but require additional or stronger ionization mechanisms. Ratios of the densities of different ion species reveal an overabundance of carbonaceous material and an underabundance of $\mathrm{H}_{2}+$ compared to the predictions of the Schmidt et al. model. While the densities of solar wind ions $\left(\mathrm{H}^{+}\right.$and $\mathrm{He}^{++}$) changed sharply across a magnetic discontinuity located $1.35 \times 10^{5} \mathrm{~km}$ from the comet, this feature, which has been called both the "cometopause" and the "magnetic pileup boundary" was barely distinguishable in the density profiles of hot cometary ions. This result is consistent with the interpretation that the magnetic pileup boundary detected by Giotto was caused by a discontinuity in the solar wind athet and is not an intrinsic feature of the interaction of the solar wind with an active comet.

Subject headings: comets -- plasmas -- abundances 


\section{INTRODUCTION}

Most cometary plasma falls into one of two categories: (1) Hot plasma consisting of material ionized in the upstream solar wind or in the slower, mass-loaded solar wind in the outer coma. These pickup ions spiral around the magnetic field carried by the solar wind and have a "thermal" speed nearly equal to the speed of the wind at their point of pickup. (2) Cold plasma in the inner coma in collisional equilibrium with the neutral gas flowing out from the nucleus with speed $<\sim 1 \mathrm{~km} / \mathrm{s}$ and a temperature of $\sim 300 \mathrm{~K}$ (Lämmerzahl et al., 1987).

The ion mass spectrometer (IMS) flown through the coma of P/Halley by the Giotto spacecraft in March, 1986, used separate sensors to study hot and cold ions. Preliminary estimates of the densities of different ion species observed by the IMS along the spacecraft track were reported by Balsiger et al. (1986, 1987a), Schwenn et al. (1987), and Balsiger (1990). The significance of those ion measurements has been interpreted by Balsiger et al. (1986), Allen et al. (1987), Geiss (1987), and Ip (1989a), among others. The intervening four years have allowed detailed reexamination of the IMS data together with more realistic analysis and modeling of the instrument's performance. It is the purpose of this paper to present the results of the recomputation of the densities of the hot component of the ion population observed by the IMS. The recomputed densities are generally greater than those presented before. The analysis has also been extended to include more ion species and finer temporal or spatial resolution. Comparison of the hot ion densities and abundances with ije predictions of a numerical physical-chemical model by Schmidt et al. (1988) allows some conclusions to be drawn about the dynamics and chemistry of the coma as well as the composition of the volatile material in the nucleus. The comparison shows that some modifications of the model are clearly required. 
Revised analyses of the hot-ion dynamics (i.e., distribution functions, velocities, and temperatures) and of the cold-ion densities and abundances will be presented in future papers.

\section{INSTRUMENT DESCRIPTION}

The IMS had two sensors, named the high-intensity spectrometer (HIS) and the high-energy-range spectrometer (HERS). HIS was optimized for measuring the cold, slow ion flow in the inner coma, whereas HERS was optimized for measuring the hot plasma in the outer coma and in the solar wind. Details of these sensors have been described previously (Balsiger et al., 1987b); only those characteristics pertinent to the discussions of data which follow are repeated here. HIS and HERS both used combinations of electric and magnetic analyzers to map out the three-dimensional velocity distributions as a function of the mass/charge ratio of the ions. Functionally, they differed in that the HIS field of view pointed along the spacecraft spin axis, which was aligned with the velocity vector of the spacecraft relative to the comet, while the HERS field of view extended from $\sim 15^{\circ}$ to $\sim 75^{\circ}$ from the spin axis. Thus HIS analyzed the dense, slow-moving plasma scooped up by the spacecraft motion, while HERS was sensitive to the hot pickup ions gyrating around the magnetic field. The present paper concentrates on the hot ion data acquired by HERS.

HERS measured ion mass/charge and velocity distributions in a $3^{\circ} \times 60^{\circ}$ fanshaped field of view which swept out a $360^{\circ}$ by $60^{\circ}$ annulus as the spacecraft spun with a period of 4 seconds. The energy/charge range of the sensor extended from $10 \mathrm{eV} / \mathrm{e}$ to a mass-dependent upper limit of $\sim 4 \mathrm{keV} / \mathrm{e}$. The sensor mass/charge range changed once per spin period, cycling through four measurement modes: the light mode with $\mathrm{m} / \mathrm{q}=2-4$ $\mathrm{amu} / \mathrm{e}$, the medium mode with $\mathrm{m} / \mathrm{q}=12-26 \mathrm{amu} / \mathrm{e}$, the heavy mode with $\mathrm{m} / \mathrm{q}=15-35$ amu/e, and the proton mode. The time to repeat observations in a given look direction for a 
given mass/charge was thus $16 \mathrm{~s}$, which corresponds to a spatial resolution along the spacecraft trajectory of $1100 \mathrm{~km}$. In the inner coma, less than $62,000 \mathrm{~km}$ from the nucleus, priority was given to HIS measurements with the consequence that two spins of HERS data were summed for each mass/charge range, thereby changing the time resolution to $32 \mathrm{~s}$ (2200 km spatial resolution). Except for the proton mode, HERS registered ions on a microchannelplate $(\mathrm{MCP})$ detector, measuring the energy/charge (64 quasilogarithmic bins), azimuth angle (64 bins denoting the phase of the spin motion), elevation angle relative to the spin axis ( 8 bins, each $7.5^{\circ}$ wide), and mass/charge (denoted by which of 40 mass anodes of the MCP recorded the ion). The output data thus formed a time series of 4dimensional arrays (counts versus energy/charge, azimuth, elevation, and mass/charge). Proton data were acquired slightly differently; their mass/charge was uniquely determined, there were only four elevation bins (each $15^{\circ}$ wide), and they were detected by channel electron multipliers rather than the MCP.

\section{METHOD OF CALCULATING DENSITIES}

The orientation of the spacecraft spin axis and velocity vector allowed the HERS to observe the solar wind for several days before the encounter with the comet. Beginning $\sim 12 \times 10^{6} \mathrm{~km}$ from the comet, HERS detected picked-up cometary protons; the cometary proton density upstream of the bow shock $\left(1.14 \times 10^{6} \mathrm{~km}\right.$ from the nucleus) has been reported by Neugebauer et al. (1989). Once inside the bow shock, the distributions of picked-up cometary protons and the heated solar wind ions overlapped in velocity space, so only the total (cometary plus solar) proton density could be calculated. The light-mode HERS data provided information on helium and other minor ion species in the solar wind, both upstream and downstream of the Halley bow shock. 
The 4-kV upper limit of energy/charge and the background counting rates of its microchannelplate (MCP) sensor prevented HERS from detecting the pickup of heavier cometary ions until the spacecraft was $\sim 250,000 \mathrm{~km}$ from the nucleus. A measurable flux of hot, cometary ions was then observed along the inbound Giotto trajectory until the spacecraft reached a distance of $40,000 \mathrm{~km}$. The plasma seen in the ram direction by HIS started to increase slowly at $150,000 \mathrm{~km}$; by $50,000 \mathrm{~km}$ most of the plasma flux was limited to the HIS field of view. Near closest approach to the nucleus, the HERS ceased operation following a severe electrical disturbance on-board the spacecraft, probably caused by a dust impact near closest approach to the nucleus; thus no HERS data were obtained on the outbound leg of the Giotto trajectory.

The calculation of cometary ion densities from the HERS data proceeded as follows:

1. The data were summed over a sufficient number of spacecraft spins to obtain $\sim 1000$ counts of water-group ions ( $\mathrm{m} / \mathrm{q}=16$ to $18 \mathrm{amu} / \mathrm{e}$, using both medium- and heavy-mode data). The lengths of these summation intervals ranged from 128 to 512 seconds, which corresponds to summing over 8 to 32 consecutive spectra for each instrument mode.

2. The count-rate matrices were corrected for detector background counts. The background count-rate corrections, which depended on both elevation angle and mass anode, were determined by averaging several hours of data obtained earlier on the day of the comet encounter when the spacecraft was millions of $\mathrm{km}$ from the nucleus and HERS could detect only solar wind ions and picked-up cometary protons. Figure 1 shows the distribution of counts versus mass anode number summed over the two elevation-angle bins closest to the ram direction (i.e., elevation angles of 15 to $30^{\circ}$ ) for the entire interval 220,000 to $40,000 \mathrm{~km}$. The spectra for the medium- and heavy-mode data are displayed 
separately, in both linear and logarithmic formats to bring out different features of the data. For the elevation bin closest to the ram direction, the MCP had a "hot spot" at the position of anode 29 , which resulted in a very high and very irregular background counting rate for that anode. Due to the time varying nature of the hot spot, the background rate for that anode was overestimated for the interval displayed in Figure 1. Data from anode 29 were not used in the analysis presented below. The numbers printed over the count-rate peaks in Figure 1 indicate the corresponding values of $\mathrm{m} / \mathrm{q}$. The count-rate versus mass anode spectra cannot be converted directly into density or flux without detailed modelling of the instrumental response; the most important factor is that the geometric factor of the instrument varied approximately as $(\mathrm{m} / \mathrm{q})^{-4}$.

3. For each of the time intervals selected in Step 1, the vector velocity of the ions with $\mathrm{m} / \mathrm{q}=16-18 \mathrm{amu} / \mathrm{e}$ was obtained by finding the least-squares fit of the observed distribution to a spherically symmetric distribution in velocity space. This process corrected for those parts of the ion distributions outside the HERS field of view. For the part of the distribution that was within the HERS field of view, the data show that isotropy is indeed a good first approximation.

4. The distribution of counts versus mass anode was then modeled using generalized Gaussians to determine the position and width of each mass peak. Examination of the flight data showed that the locations of the mass peaks had shifted by a fraction of a mass anode in the year between laboratory calibration of the instrument and the comet encounter. The cause of this shift is not understood. Thus the calibration data were used as a guide to the approximate locations and widths of the mass peaks and least-squares fits of the flight data were performed to determine the best values to use for the data analysis. It was assumed that neither the locations nor the widths of the mass peaks changed during the hour before closest approach to the comet. The positions and shapes of each of the mass 
peaks were determined for the data set as a whole, while the contributions of different ion species to the counts measured by each anode were separately determined for each time interval by performing a least-squares analysis to determine the height of each peak. Although it was originally intended to use both the medium- and heavy-mode data for calculating the densities of water-group ions, the least-squares fits to the medium-mode data were significantly superior to the heavy-mode data fits (the variances between the data and the fits were smaller and the peak widths determined by the fits were closer to the prelaunch calibration values), so only the medium-mode data were used to calculate the densities for $\mathrm{m} / \mathrm{q}=16-18 \mathrm{amu} / \mathrm{e}$. Figure 2 illustrates the type of fit that could be obtained; it shows the measured water-group counts in anodes 18-27 for medium-mode measurements. In this example, the measured counts are indicated by circles with error bars representing the uncertainty arising from counting statistics; the upper curve shows the least-squares fit; while the dotted curves show the individual contributions of ions with $\mathrm{m} / \mathrm{q}$ $=16,17$, and $18 \mathrm{amu} / \mathrm{e}$.

5. For each interval, it was then assumed that each ion species had an isotropic distribution and the same bulk velocity vector $\mathbf{v}_{\mathbf{0}}$ as the water-group ions (as determined in Step 3). Then, for each value of $\mathrm{m} / \mathrm{q}$, the 3-D distribution in elevation-azimuthenergy/charge was transformed into a one-dimensional distribution of phase space density versus $|\Delta v|$, where $\Delta v=v-v_{0}$. Integration over this 1-D distribution then gave the ion density.

The method used for computing proton densities was similar except that:

1. The proton counting rate was high enough that the proton density could be calculated on a spin by spin basis to yield $1100 \mathrm{~km}$ resolution. The computed proton densities have been averaged over 6 minutes $(25,000 \mathrm{~km})$ for the purposes of this paper, but the full 
resolution data have been submitted to the Intemational Halley Watch (IHW) and the US National Space Science Data Center (NSSDC) archives.

2. A moments technique, rather than a least-squares fit was used to calculate the proton bulk velocity independent of the heavy-ion velocity calculated in Step 2 above.

3. The HERS instrument was able to identify protons unambiguously, with no mass overlap, so Step 4 above was not necessary.

The fluxes of ions with $\mathrm{m} / \mathrm{q}=2 \mathrm{amu} / \mathrm{e}$ were great enough to allow $64 \mathrm{sec}(4400$ $\mathrm{km})$ resolution. As for the protons, 6-minute $(25,000 \mathrm{~km})$ averages are presented in this paper, with the full resolution data available from the authors or through the IHW or NSSDC.

\section{RESULTS}

The density values computed as described above are listed in Tables 1-13 -- one table for each value of mass/charge. Each table includes a quality index, which ranges from 1 to 5 . These indices should be interpreted as follows:

Quality $=1$ : high count rate and negligible statistical uncertainty. Also no significant overlap with any other mass peak. The only important uncertainty in the density is the absolute calibration of the instrument, which is estimated to be $\sim \pm 30 \%$. Only protons and ions with $\mathrm{m} / \mathrm{q}=2$ and $12 \mathrm{amu} / \mathrm{e}$ were assigned Quality $=1$.

Quality $=2$ : high count rate and negligible statistical uncertainty, but $\sim 10 \%$ additional uncertainty due to overlapping mass peaks, leading to a total uncertainty of $\left(.30^{2}+.10^{2}\right)^{1 / 2}$ $=32 \%$. Water-group ions $(\mathrm{m} / \mathrm{q}=16-18 \mathrm{amu} / \mathrm{e})$ fall in this category. 
Quality = 3: low count rate with an additional uncertainty of $\sim 10 \%$ due to counting statistics (32\% total uncertainty), but no significant problem due to mass-peak overlaps. Ions with mass/charge 14 and 32 fall in this category despite the use of longer averaging intervals for those ion species.

Quality $=4:$ moderate count rates and severe problems of overlap with a neighboring mass peak. The additional uncertainty is $\sim 25 \%$ (39\% total uncertainty). Ions with mass $/$ charge $=28 \mathrm{amu} / \mathrm{e}$ have Quality $=4$.

Quality $=5$ : severe problems with low count rates and/or a large neighboring mass peak. The additional uncertainty may be $35 \%$, to yield a total uncertainty of $46 \%$. Ions with Quality $=5$ are at $4,13,15$, and $29 \mathrm{amu} / \mathrm{e}$.

The count-rate spectra in Figure 1 show some evidence for minor ion species for which we have not presented densities. There was clearly some small number of ions with $\mathrm{m} / \mathrm{q}=19 \mathrm{amu} / \mathrm{e}$ present. For the more reliable medium-mode data, the mass/charge peak for $\mathrm{m} / \mathrm{q}=19 \mathrm{amu} / \mathrm{e}$ straddled the hot spot on anode 29 , and it showed up as only a small shoulder on the heavy-mode $\mathrm{m} / \mathrm{q}=18 \mathrm{amu} / \mathrm{e}$ peak. The HIS data showed that although ions with $\mathrm{m} / \mathrm{q}=19 \mathrm{amu} / \mathrm{e}$ (presumably mostly $\mathrm{H}_{3} \mathrm{O}^{+}$) were the dominant species at cometocentric distances $<2000 \mathrm{~km}$, they provided less than $10 \%$ of the cold ion density outside $35,000 \mathrm{~km}$ and less than $1 \%$ outside $80,000 \mathrm{~km}$. We conclude that $\mathrm{H}_{3} \mathrm{O}^{+}$is an important species only in the collision dominated inner coma.

Figure 1 also shows several small peaks between $\mathrm{m} / \mathrm{q}=19$ and $28 \mathrm{amu} / \mathrm{e}$. None of these peaks was more than 2 standard deviations above background. Further work on limiting the region of phase space examined may allow calculation of a density for the $\mathrm{m} / \mathrm{q}$ 
$=24 \mathrm{amu} / \mathrm{e}$ peak which had counts in two adjacent anodes in both the medium and heavy modes.

It must be emphasized that inside $80,000 \mathrm{~km}$ the densities given in Tables 1-13 are lower limits to the total ion densities in that they refer only to the hot ions whose velocity distributions overlapped the field of view of the HERS sensor. They are the ions picked up by the mass-loaded solar wind which, at cometocentric distances $>40,000 \mathrm{~km}$, still had a flow speed exceeding $10 \mathrm{~km} / \mathrm{s}$ relative to the comet nucleus. As the spacecraft approached the comet, the density of the hot ions reached a maximum of $-150 \mathrm{~cm}^{-3}$ near $75,000 \mathrm{~km}$, and then declined as the hot ions were replaced by cold ions due to collisions with the cometary neutral gas. The hot-ion population was too small to be detected by the IMS by the time the spacecraft reached $40,000 \mathrm{~km}$. This is consistent with the findings of other instruments on Giotto; Krankowsky et al. (1986), for example, reported that evidence for energetic ions persisted in to $\sim 40,000 \mathrm{~km}$ from the nucleus, and Korth et al. (1987) reported high temperatures and broad ion distributions outside $43,000 \mathrm{~km}$. Density or flux profiles of the cold ions in the inner coma have been published by Balsiger et al. (1986, 1987a), Balsiger (1990), Schwenn et al. (1987), Kettmann et al. (1990), Korth et al. (1987), and Krankowsky et al. (1986).

The circles and diamonds in Figure 3 show the 6-minute average densities of protons and ions with $m / q=2 \mathrm{amu} / \mathrm{e}$, respectively, from Tables 1 and 2 plotted versus distance from the comet. Although inside $200,000 \mathrm{~km} \mathrm{H}_{2}{ }^{+}$contributed to the density of ions with $\mathrm{m} / \mathrm{q} \sim 2 \mathrm{amu} / \mathrm{e}$ (Fuselier et al., 1988), at greater distances the $\mathrm{m} / \mathrm{q}=2 \mathrm{amu} / \mathrm{e}$ population consisted almost entirely of $\mathrm{He}^{++}$ions from the solar wind. The measured number-density ratio $n_{2} / n_{1}$ ranged from 0.02 to 0.04 , which is quite typical for the lowspeed solar wind near interplanetary sector boundaries, such as that observed at the Giotto encounter with P/Halley. Fuselier et al. (1990) consider the ion abundances in the solar 
wind and the charge-exchange reactions of solar wind ions with the coma gas in much greater detail. The continuous curve shown in this figure is disussed in the following section. The jump in proton and $\mathrm{He}^{++}$densities at the Halley bow shock is indicated by the vertical line at $1.14 \times 10^{6} \mathrm{~km}$.

The dashed vertical line in Figure 3 at $1.35 \times 10^{5} \mathrm{~km}$ marks the crossing of a discontinuity that has been called both the "cometopause" and the "magnetic pileup boundary". Whatever its proper name, its most striking feature was a sudden jump in the strength of the magnetic field from $\sim 10$ to $-30 \mathrm{nT}$ (Neubauer et al., 1986). It is clear that on the scale of Figure 3 (one point per $25,000 \mathrm{~km}$ ), the magnetic pileup boundary marked a sharp decrease in the density of solar-wind ions.

In Figure 4, the circular symbols repeat portions of the proton and $\mathrm{m} / \mathrm{q}=2 \mathrm{amu} / \mathrm{e}$ data from Figure 3 and also show the densities of the other ion species listed in Tables 413. The principal difference between the formats of Figures 3 and 4 is that in Figure 4 the distance scale is logarithmic and corresponds to a smaller range -- from 25,000 to 250,000 $\mathrm{km}$ (log distances $=4.4-5.4$, respectively). The dashed vertical line again locates the magnetic pileup boundary; the bow-shock would be located off-scale to the right. Again, discussion of the continuous curves is postponed to the following section.

The distance profiles of the cometary ions $(\mathrm{m} / \mathrm{q}=12-32 \mathrm{amu} / \mathrm{e})$ were markedly different from those of the solar wind ions. The density of each species of hot cometary ion reached a maximum between 60,000 and $100,000 \mathrm{~km}(\log$ distance $=4.8-5.0)$, and each of their distance profiles had roughly the same shape. Inside the maxima, the hot ions were removed by collisions. Outside the maxima, the densities of the hot cometary ions continued to decrease with increasing distance, barely reacting to the magnetic pileup boundary at all. 
Figure 5 displays several ratios of ion densities as a function of distance from the comet in the same format as Figure 4. Again, please ignore the continuous curves until the next section. The increases in the ratios $n_{16} / n_{18}, n_{17} / n_{18}$, and $n_{12} /\left(n_{28}+n_{29}\right)$ with distance and the decrease in $n_{13} / n_{12}$ are consistent with expectations of the breakup of molecules into smaller molecules and atoms. The principal reason for the steep rise in $n_{2} / \mathrm{n}_{18}$ with distance is the increasing amount of solar wind $\mathrm{He}^{++}$.

The horizontal line in the panel displaying the ratio $n_{13} / n_{12}$ indicates the value of the ratio that would be observed if all the $\mathrm{m} / \mathrm{q}=13 \mathrm{amu} / \mathrm{e}$ ions were ${ }^{13} \mathrm{C}^{+}$and if the ratio of ${ }^{12} \mathrm{C} /{ }^{13} \mathrm{C}=63$, as reported by Wyckoff and Lindholm (1989) from ground-based observations of ${ }^{13} \mathrm{C}^{14} \mathrm{~N}$ in comet Halley. The observed ratio is above that line, which indicates the presence of $\mathrm{CH}^{+}$ions.

\section{COMPARISON TO THE THEORETICAL MODEL OF SCHMIDT ET AL.}

The most ambitious model of the physics and chemistry of cometary ions is that of Schmidt et al. (1988); we shall hereafter use the acronym SWHB to refer to that paper and model. SWHB produced hydrodynamic and magnetohydrodynamic simulations of the gas and plasma flow around Halley's comet with a detailed photo and chemical reaction network (19 different processes) of 59 neutral and 76 ionized chemical species. Their model assumed that all cometary gas was released directly from the nucleus and did not include dust which is known to be a distributed source of gas (e.g., Eberhardt et al., 1987). The model assumes a composition of $80.0 \% \mathrm{H}_{2} \mathrm{O}, 8.0 \% \mathrm{CO}, 3.0 \% \mathrm{CO}_{2}, 2.0 \%$ $\mathrm{CH}_{4}, 2.0 \% \mathrm{NH}_{3}, 2.0 \% \mathrm{H}_{2} \mathrm{CO}, 1.0 \% \mathrm{CS}_{2}, 0.68 \% \mathrm{~N}_{2}, 0.67 \% \mathrm{C}_{2} \mathrm{H}_{2}, 0.05 \% \mathrm{H}_{2} \mathrm{C}_{3} \mathrm{H}_{2}$, $0.30 \% \mathrm{H}_{2} \mathrm{CO}_{2}, 0.16 \% \mathrm{CH}_{3} \mathrm{CN}, 0.08 \% \mathrm{NH}_{2} \mathrm{CH}_{3}$, and $0.06 \% \mathrm{HCN}$. SWHB calculated the densities of different ion species expected along the Giotto trajectory, using gas 
production rates and solar wind parameters appropriate to the time of the Giotto encounter with $\mathrm{P} / \mathrm{Halley}$. Their densities and density ratios are indicated by the continuous curves in Figures 3 through 5 .

From Figure 3, it can be seen that outside the magnetic pileup boundary the observed proton density profile is in fairly good agreement with the SWHB model. The disagreement inside the magnetic pileup boundary can be qualitatively accounted for by the fact that the SWHB curve represents the total (both hot and cold) proton density. Although the SWHB model shows substantial cooling of the ions inside $10^{5} \mathrm{~km}$, it has no discontinuous jump in temperature anywhere between the contact surface (also called the ionopause or the diamagnetic cavity boundary) observed at $4700 \mathrm{~km}$ (Neubauer et al., 1986) and the bow shock. The calculated position of the bow shock (slightly beyond the end of the curve plotted in Figure 3) is slightly farther from the comet than was the observed shock.

The center panel in the top row of Figure 4 compares the model to the observed densities of ions with $m / q=2 \mathrm{amu} / \mathrm{e}$. SWHB did not include solar-wind helium ions in their model; this explains why the model profile drops while the observed density rises with increasing distance. In SWHB, ions with $\mathrm{m} / \mathrm{q}=2 \mathrm{amu} / \mathrm{e}$ are entirely $\mathrm{H}_{2}{ }^{+}$, while the observations show a mixture of $\mathrm{H}_{2}{ }^{+}$and $\mathrm{He}^{++}$with an increasing proportion of $\mathrm{H}_{2}{ }^{+}$closer to the comet (Fuselier et al., 1988). What is interesting is that inside the magnetic pileup boundary, the sum of the observed $\mathrm{H}_{2}{ }^{+}$and $\mathrm{He}^{++}$is less than the SWHB density for $\mathrm{H}_{2}{ }^{+}$ alone.

The remaining panels in Figure 4 show that all other ion species had significantly higher densities than predicted by the simulation, The differences often exceed an order of magnitude. The observational profiles are generally much more concave downward than 
are the SWHB profiles. At small distances, the difference is caused by collisional cooling of the hot ions which removes them from the HERS field of view. For many species (i.e., $\mathrm{m} / \mathrm{q}=16,17,18,28$, and $29 \mathrm{amu} / \mathrm{e}$ ) the observed slope at large distance is steeper than the slope predicted by SWHB; no simple reason for this discrepancy is apparent. Outside the magnetic pileup boundary, each of these species dropped off more rapidly than $r^{2}$.

Figure 5 shows excellent agreement between the data and the SWHB model for the ratios $n_{16} / n_{18}$ and $n_{17} / n_{18}$, indicating that the simulation does a good job of modeling the dissociation and ionization of water molecules and their products. The ratio of $n_{12} / n_{16}$ indicates that the model underestimates the relative amount of carbonaceous material. The fact that the observed value of the ratio $n_{12} /\left(n_{28}+n_{29}\right)$ is greater than the model value of this ratio probably indicates that the missing carbonaceous material is not entirely additional CO. The SWHB model does not include any ${ }^{13} \mathrm{C}$, which can account for some of, but not all the mismatch between the observed and calculated values of $n_{13} / n_{12}$. Because the photodissociation lifetime of $\mathrm{CH}$ molecules is only $100 \mathrm{~s}$, the source of the $\mathrm{CH}^{+}$is not obvious.

The ratio $n_{14} / n_{16}$ is also higher than that given by SWHB, by about the same factor as the $\mathrm{n}_{12} / \mathrm{n}_{16}$ ratio. The ion peak at $\mathrm{m} / \mathrm{q}=14 \mathrm{amu} / \mathrm{e}$, however, has contributions from both $\mathrm{N}^{+}$and $\mathrm{CH}_{2}^{+}$, so it is not safe to conclude that the comet must have more nitrogenbearing material than was included in the SWHB model.

\section{DISCUSSION}

HERS did detect most of the ions expected to be found in the coma of comet Halley. The HERS mass/charge spectra had peaks corresponding to each of the ions detected spectroscopically from Earth $\left(\mathrm{C}^{+}, \mathrm{CH}^{+}, \mathrm{NH}^{+}, \mathrm{OH}^{+}, \mathrm{H}_{2} \mathrm{O}^{+}, \mathrm{CN}^{+}, \mathrm{CO}^{+}, \mathrm{N}_{2}^{+}\right.$, 
$\mathrm{Ca}^{+}$, and $\mathrm{CO}_{2}^{+}$) except for those heavier than the $35 \mathrm{amu} / \mathrm{e}$ range of the instrument. It was no surprize that water-group ions were found to be the most abundant. It is, perhaps, surprizing that the densities of both $\mathrm{Na}^{+}$and $\mathrm{C}_{2}{ }^{+}$were below the detection threshold, especially in light of the observation of a major peak at $m / q=23-24 \mathrm{amu} / \mathrm{e}$ in the ion spectra obtained by the ICE spacecraft at P/Giacobini-Zinner (Ogilvie et al., 1986). Ip (1989b) has argued that the expected density of $\mathrm{Na}^{+}(\mathrm{m} / \mathrm{q}=23 \mathrm{amu} / \mathrm{e})$ is $>10^{-3}$ times the density of water-group ions, which is close to the detection limit of HERS. Detection of ions with $\mathrm{m} / \mathrm{q}=23$ and $24 \mathrm{amu} / \mathrm{e}$ in the inner coma (i.e., in the cold-ion region) of $\mathrm{P} / \mathrm{Halley}$ has been reported by Krankowsky et al. (1986) and Eviatar et al. (1989).

One of the principal conclusions of the present study is that the density of cometary ions in the distance range 40,000 to $250,000 \mathrm{~km}$ is at least an order of magnitude higher than the predicted density. This discrepancy had previously been noted by Ip (1989a) on the basis of preliminary values of ion density. Furthermore, the problem is not unique to the SWHB model. For example, the calculation by Ip (1989b) shows a similar disparity The density of protons is, however, in good agreement with the models. Thus, we can rule out compression of the plasma as the cause of the high density of cometary ions, because the solar-wind protons would have been compressed too. Another argument against significant compression is that the strength of the magnetic field was significantly less than predicted by the model outside the magnetic pileup boundary and roughly the same as the model field inside it (Huebner et al., 1989).

Ip (1989b) and Marconi and Mendis (1988) have suggested that the solar EUV flux at the time of the Giotto encounter may have been significantly greater than the typical solar-minimum values assumed in the models. But Ip (1989b) has argued that even an order-of-magnitude increase in solar EUV would not lead to an order-of-magnitude increase in the ionization rate because of the importance of other mechanisms for creating 
cometary ions, such as charge exchange with the solar wind. Furthermore, there is not necessarily a direct relation between ion density and ionization rate.

Perhaps the models underestimate the rate of ionization associated with chargeexchange reactions. The results of the study by Shelley et al. (1987) were consistent with an anomalously high charge-exchange rate of $\mathrm{He}^{++}$. There are several effects associated with charge exchange that have not been included in the models. (1) The velocity dependence of the charge-exchange cross sections was neglected, but it is known that the cross sections increase with decreasing velocity. (2) The SWHB model does not include ionization by or other effects of the fast neutral products of charge-exchange interactions. Ip (1990) has shown, however, that energetic neutral atoms can play an important role in the physics of the coma. (3) In the models, the term accounting for the source of cometary ions due to charge exchange is appropriate for an unmagnetized plasma. It does not include the greater path length traversed by a hot ion which gyrates around the magnetic field. (4) The flow field and the relative velocity between the solar wind and cometery neutrals may differ from that in the models. A comparision of the observed velocities with those predicted by various models is the topic of a future study.

There may have been other sources of "anomalous ionization", which was a topic of great interest many years ago. For example, tail currents closing through the inner coma could be an important source of ionization; Ip (1979) has drawn an analogy with energy deposition during terrestrial substorms. Along this line, Neubauer (1988) suggested that a possible cause of a weak shock observed by the Giotto magnetometer to be propagating radially outward at a distance of $\sim 5000 \mathrm{~km}$ might have been caused by a transient injection of hot ions and electrons following magnetic merging in the tail. There are also other spacecraft observations that have been interpreted as evidence for field-line reconnection on the dayside of Halley's coma (Verigin et al., 1987; Kirsch et al., 1989). It is questionable 
whether such processes could contribute to the level of ionization on the large scale observed.

Another type of anomalous ionization that has been suggested in connection with comets is the critical ionization velocity (CIV) effect (Formisano et al., 1982; Galeev et al., 1986). In this effect, first postulated by Alfven (1954), the kinetic energy of the relative flow of a neutral gas across magnetic field lines is collisionlessly transferred to the plasma electrons which then collide with and ionize the gas. The difficulty with invoking CIV to explain the anomalous ionization observed by HERS is the absence of a simultaneous large flux of energetic electrons. The RPA instrument on Giotto had a $10-\mathrm{eV}$ threshold for detecting electrons; it did detect anomalously high fluxes of $\mathrm{keV}$ electrons in the so-called "mystery region" at distances from the nucleus between 550,000 and 850,000 km (Reme et al., 1987), but the HERS energy range and sensitivity threshold prevented observations of cometary ions at those distances. The RPA investigators have estimated that even in the sharpest spikes of energetic electron fluxes observed in the mystery region, the rate of ionization due to electron impact was less than twice the photoionization rate (R. P. Lin, personal communication). Such relatively weak and localized sources of ionization probably cannot account for the higher than expected ion densities.

It also cannot be ruled out that temporal variation or anisotropic emission of gas from the comet might have contributed to the excess ionization. Approximately 1 day is required for the neutral gas to travel each $10^{5} \mathrm{~km}$ from the nucleus. Thus the gas and ions observed at $2 \times 10^{5} \mathrm{~km}$ left the nucleus approximately a day earlier than the gas and ions detected at $1 \times 10^{5} \mathrm{~km}$. IUE observations by Feldman et al. (1987) showed a 25\% decrease in brightness during the day prior to the Giotto closest approach to P/Halley. The groundbased observations by Millis and Schleicher (1986), on the other hand, showed very little change in the comet's gas production rate over this same interval. 
The ratios of the densities of different ion species put important constraints on cometary chemistry. Comparison of the density ratios $n_{16} / n_{18}$ and $n_{17} / n_{18}$ to ratios computed from the SWHB model might suggest that the major chain of dissociation and ionization of water is well understood. One major discrepancy between observations and the model is the overabundance of $\mathrm{C}^{+}$and the higher than predicted ratios of $n_{13} / n_{12}$, $n_{14} / n_{16}$, and $n_{12} /\left(n_{28}+n_{29}\right)$. One suspects that the extra carbonaceous material probably originated in the CHON grains (Kissel et al., 1986) which were a distributed source of gas in the coma (Eberhardt et al., 1987), whereas SWHB did not include any source other than the comet's surface. The underabundance of $\mathrm{H}_{2}{ }^{+}$compared to the model is a much more difficult problem which requires detailed chemical modeling to understand.

Comparison of the SWHB model and the HERS data shows that the model requires iteration before the molecular composition of the volatiles in the nucleus can be deduced. The discrepancy seems to be both of a physical and chemical nature because neither the total nor the relative ion abundances predicted by the model fit the data.

Finally, we wish to comment on the implications of the HERS density data for the nature of the magnetic pileup boundary. That feature was marked by sudden changes in the density of solar wind protons and alphas, in the strength of the magnetic field, and in the density of electrons with energy $>10 \mathrm{eV}$ (Reme et al., 1987). Its magnetic structure was consistent with that of a tangential discontinuity (Neubauer, 1987). As pointed out previously (Balsiger et al., 1987a; Balsiger, 1990; d'Uston et al., 1987), it was not accompanied by a discontinuous change in either the density or the chemical composition of hot cometary ions. Thus it was not a "chemical boundary" as proposed by Gringauz et al. (1986) on the basis of observations of a discontinuity in a similar region of the coma by the Vega spacecraft. The HERS data support the conclusion of Raeder et al. (1989) that the 
magnetic pileup boundary observed by Giotto was not an intrisic feature of the interaction of an active comet with the solar wind, but rather a response to the passage of an interplanetary discontinuity of a type commonly observed near magnetic sector boundaries in the solar wind.

Acknowledgements. We thank $\mathrm{R}$. Wegmann for providing tables of the model ion densities along the Giotto trajectory in computer readable format. We also thank all members of the IMS team at Lockheed Palo Alto Research Laboratory, the University of Bern, and the Max-Planck-Institut für Aeronomie who made this investigation possible. The work at the Jet Propulsion Laboratory was done under a contract between the Califomia Institute of Technology and the National Aeronautics and Space Administration under the sponsorship of the Planetary Atmospheres program. Research at Lockheed was supported by NASA through contract NASW-4336. This work was also supported by the Swiss National Science Foundation and by the German Bundesministerium für Forschung und Technologie. 
TABLE 1. $\mathrm{m} / \mathrm{q}=1$. (Quality $=1$ )

\begin{tabular}{|c|c|c|}
\hline Decimal Hrs & Distance $(\mathrm{km})$ & $\begin{array}{l}\text { Density } \\
\left(\mathrm{cm}^{-3}\right)\end{array}$ \\
\hline 18.550 & $0.135 \mathrm{E}+07$ & 6.90 \\
\hline 18.650 & $0.133 E+07$ & 7.14 \\
\hline 18.750 & $0.130 E+07$ & 7.75 \\
\hline 18.900 & $0.127 E+07$ & 7.83 \\
\hline 19.050 & $0.123 E+07$ & 8.13 \\
\hline 19.150 & $0.121 E+07$ & 8.30 \\
\hline 19.250 & $0.118 \mathrm{E}+07$ & 7.20 \\
\hline 19.350 & $0.116 E+07$ & 6.59 \\
\hline 19.450 & $0.113 E+07$ & 9.18 \\
\hline 19.550 & $0.111 \mathrm{E}+07$ & 10.07 \\
\hline 19.650 & $0.108 E+07$ & 10.36 \\
\hline 19.750 & $0.106 E+07$ & 12.09 \\
\hline 19.850 & $0.103 E+07$ & 11.48 \\
\hline 19.950 & $0.101 E+07$ & 11.80 \\
\hline 20.050 & $0.985 E+06$ & 12.43 \\
\hline 20.150 & $0.960 \mathrm{E}+06$ & 10.64 \\
\hline 20.250 & $0.935 E+06$ & 10.08 \\
\hline 20.350 & $0.911 E+06$ & 9.64 \\
\hline 20.450 & $0.886 E+06$ & 13.34 \\
\hline 20.550 & $0.861 \mathrm{E}+06$ & 16.21 \\
\hline 20.650 & $0.837 E+06$ & 15.94 \\
\hline 20.750 & $0.812 \mathrm{E}+06$ & 19.27 \\
\hline 20.850 & $0.788 \mathrm{E}+06$ & 16.73 \\
\hline 20.950 & $0.763 E+06$ & 16.93 \\
\hline 21.050 & $0.738 E+06$ & 16.44 \\
\hline 21.150 & $0.714 E+06$ & 17.52 \\
\hline 21.250 & $0.689 E+06$ & 18.15 \\
\hline 21.350 & $0.665 E+06$ & 16.43 \\
\hline 21.450 & $0.640 \mathrm{E}+06$ & 13.59 \\
\hline 21.550 & $0.615 E+06$ & 14.42 \\
\hline 21.650 & $0.591 \mathrm{E}+06$ & 14.47 \\
\hline 21.750 & $0.566 E+06$ & 13.52 \\
\hline 21.850 & $0.542 E+06$ & 13.04 \\
\hline 21.950 & $0.517 E+06$ & 12.67 \\
\hline 22.050 & $0.492 \mathrm{E}+06$ & 13.02 \\
\hline 22.150 & $0.468 E+06$ & 13.75 \\
\hline 22.250 & $0.443 E+06$ & 13.63 \\
\hline 22.350 & $0.418 E+06$ & 13.45 \\
\hline 22.450 & $0.394 E+06$ & 13.07 \\
\hline 22.550 & $0.369 E+06$ & 11.99 \\
\hline 22.650 & $0.345 E+06$ & 11.14 \\
\hline 22.750 & $0.320 E+06$ & 9.23 \\
\hline 22.850 & $0.295 E+06$ & 9.32 \\
\hline 22.950 & $0.271 \mathrm{E}+06$ & 10.12 \\
\hline 23.050 & $0.246 E+06$ & 10.63 \\
\hline 23.150 & $0.222 E+06$ & 9.93 \\
\hline 23.250 & $0.197 \mathrm{E}+06$ & 8.85 \\
\hline 23.350 & $0.172 E+06$ & 7.94 \\
\hline 23.450 & $0.148 E+06$ & 6.57 \\
\hline 23.550 & $0.123 E+06$ & 2.84 \\
\hline 23.650 & $0.985 E+05$ & 3.52 \\
\hline 23.750 & $0.738 E+05$ & 2.62 \\
\hline 23.850 & $0.492 E+05$ & 0.86 \\
\hline 23.950 & $0.246 \mathrm{E}+05$ & 0.40 \\
\hline 24.000 & $0.123 E+05$ & 0.20 \\
\hline
\end{tabular}

TABLE 2. $\mathrm{m} / \mathrm{q}=2$. (Quality $=1$ )

\begin{tabular}{|c|c|c|}
\hline Decimal Hrs & Distance $(\mathrm{km})$ & $\begin{array}{l}\text { Density } \\
(\mathrm{cm}-3)\end{array}$ \\
\hline 18.550 & $0.135 E+07$ & 0.18 \\
\hline 18.650 & $0.133 E+07$ & 0.20 \\
\hline 18.750 & $0.130 E+07$ & 0.19 \\
\hline 18.900 & $0.127 E+07$ & 0.20 \\
\hline 19.050 & $0.123 E+07$ & 0.20 \\
\hline 19.150 & $0.121 E+07$ & 0.18 \\
\hline 19.250 & $0.118 E+07$ & 0.15 \\
\hline 19.350 & $0.116 E+07$ & 0.14 \\
\hline 19.450 & $0.113 E+07$ & 0.29 \\
\hline 19.550 & $0.111 E+07$ & 0.29 \\
\hline 19.650 & $0.108 E+07$ & 0.30 \\
\hline 19.750 & $0.106 E+07$ & 0.26 \\
\hline 19.850 & $0.103 E+07$ & 0.36 \\
\hline 19.950 & $0.101 E+07$ & 0.37 \\
\hline 20.050 & $0.985 E+06$ & 0.35 \\
\hline 20.150 & $0.960 E+06$ & 0.30 \\
\hline 20.250 & $0.935 E+06$ & 0.32 \\
\hline 20.350 & $0.911 E+06$ & 0.28 \\
\hline 20.450 & $0.886 E+06$ & 0.41 \\
\hline 20.550 & $0.861 E+06$ & 0.46 \\
\hline 20.650 & $0.837 E+06$ & 0.61 \\
\hline 20.750 & $0.812 E+06$ & 0.76 \\
\hline 20.850 & $0.788 E+06$ & 0.62 \\
\hline 20.950 & $0.763 E+06$ & 0.58 \\
\hline 21.050 & $0.738 E+06$ & 0.56 \\
\hline 21.150 & $0.714 E+06$ & 0.57 \\
\hline 21.250 & $0.689 E+06$ & 0.60 \\
\hline 21.350 & $0.665 E+06$ & 0.69 \\
\hline 21.450 & $0.640 E+06$ & 0.60 \\
\hline 21.550 & $0.615 E+06$ & 0.60 \\
\hline 21.650 & $0.591 E+06$ & 0.63 \\
\hline 21.750 & $0.566 E+06$ & 0.58 \\
\hline 21.850 & $0.542 E+06$ & 0.45 \\
\hline 21.950 & $0.517 E+06$ & 0.37 \\
\hline 22.050 & $0.492 E+06$ & 0.35 \\
\hline 22.150 & $0.468 E+06$ & 0.40 \\
\hline 22.250 & $0.443 E+06$ & 0.45 \\
\hline 22.350 & $0.418 E+06$ & 0.41 \\
\hline 22.450 & $0.394 E+06$ & 0.41 \\
\hline 22.550 & $0.369 E+06$ & 0.38 \\
\hline 22.650 & $0.345 E+06$ & 0.37 \\
\hline 22.750 & $0.320 E+06$ & 0.32 \\
\hline 22.850 & $0.295 E+06$ & 0.35 \\
\hline 22.950 & $0.271 E+06$ & 0.35 \\
\hline 23.050 & $0.246 E+06$ & 0.41 \\
\hline 23.150 & $0.222 E+36$ & 0.39 \\
\hline 23.250 & $0.197 E+06$ & 0.37 \\
\hline 23.350 & $0.172 E+06$ & 0.28 \\
\hline 23.450 & $0.148 E+06$ & 0.25 \\
\hline 23.550 & $0.123 E+06$ & 0.12 \\
\hline 23.650 & $0.985 E+05$ & 0.12 \\
\hline 23.750 & $0.738 E+05$ & 0.07 \\
\hline 23.850 & $0.492 \mathrm{E}+05$ & 0.04 \\
\hline 3900 & $0.369 E+05$ & 0.05 \\
\hline
\end{tabular}


TABLE 3. $\mathrm{m} / \mathrm{q}=4$ (Quality $=5$ )

\begin{tabular}{|c|c|c|c|}
\hline Decimal Hrs & Distance $(\mathrm{km})$ & $\begin{array}{c}\text { Total } \\
\text { Time }(\mathrm{s})\end{array}$ & $\begin{array}{c}\text { Density } \\
\left(\mathrm{cm}^{-3}\right)\end{array}$ \\
\hline 22.116 & $0.476 \mathrm{E}+06$ & 3150 & 0.014 \\
22.616 & $0.353 \mathrm{E}+06$ & 1800 & 0.016 \\
22.949 & $0.271 \mathrm{E}+06$ & 1200 & 0.015 \\
23.116 & $0.230 \mathrm{E}+06$ & 600 & 0.020 \\
23.283 & $0.189 \mathrm{E}+06$ & 600 & 0.015 \\
23.449 & $0.148 \mathrm{E}+06$ & 600 & 0.014 \\
23.616 & $0.107 \mathrm{E}+06$ & 600 & 0.024 \\
23.783 & $0.657 \mathrm{E}+05$ & 600 & 0.016 \\
\hline
\end{tabular}

TABLE 4. $\mathrm{m} / \mathrm{a}=12$ (Qualitv $=1$ )

\begin{tabular}{|c|c|c|c|}
\hline Decimal Hrs & Distance $(\mathrm{km})$ & $\begin{array}{c}\text { Total } \\
\text { Time }(\mathrm{s})\end{array}$ & $\begin{array}{c}\text { Density } \\
\left(\mathrm{cm}^{-3}\right)\end{array}$ \\
\hline 23.087 & $0.237 \mathrm{E}+06$ & 496 & 1.0 \\
23.227 & $0.203 \mathrm{E}+06$ & 512 & 1.1 \\
23.372 & $0.167 \mathrm{E}+06$ & 512 & 2.4 \\
23.463 & $0.144 \mathrm{E}+06$ & 128 & 3.6 \\
23.498 & $0.136 \mathrm{E}+06$ & 128 & 4.4 \\
23.534 & $0.127 \mathrm{E}+06$ & 128 & 5.0 \\
23.569 & $0.118 \mathrm{E}+06$ & 128 & 5.4 \\
23.605 & $0.110 \mathrm{E}+06$ & 128 & 5.1 \\
23.640 & $0.101 \mathrm{E}+06$ & 128 & 8.3 \\
23.676 & $0.921 \mathrm{E}+05$ & 128 & 9.3 \\
23.711 & $0.834 \mathrm{E}+05$ & 128 & 11.0 \\
23.747 & $0.746 \mathrm{E}+05$ & 128 & 10.4 \\
23.782 & $0.660 \mathrm{E}+05$ & 128 & 11.3 \\
23.823 & $0.559 \mathrm{E}+05$ & 160 & 6.6 \\
23.867 & $0.450 \mathrm{E}+05$ & 160 & 11.8 \\
\hline
\end{tabular}

TABLE 5. $\mathrm{m} / \mathrm{q}=13$ (Quality $=5$ )

\begin{tabular}{|c|c|c|c|}
\hline Decimal Hrs & Distance (km) & $\begin{array}{c}\text { Total } \\
\text { Time }(\mathrm{s})\end{array}$ & $\begin{array}{c}\text { Density } \\
\left(\mathrm{cm}^{-3}\right)\end{array}$ \\
\hline 23.534 & $0.127 \mathrm{E}+06$ & 640 & 0.1 \\
23.710 & $0.837 \mathrm{E}+05$ & 640 & 0.4 \\
23.867 & $0.450 \mathrm{E}+05$ & 480 & 0.2 \\
\hline
\end{tabular}

TABLE 6. $\mathrm{m} / \mathrm{q}=14$ (Quality $=3$ )

\begin{tabular}{|c|c|c|c|}
\hline Decimal Hrs & Distance (km) & $\begin{array}{c}\text { Total } \\
\text { Time (s) }\end{array}$ & $\begin{array}{c}\text { Density } \\
\left(\mathrm{cm}^{-3}\right)\end{array}$ \\
\hline 23.300 & $0.185 \mathrm{E}+06$ & 1056 & 0.3 \\
23.534 & $0.127 \mathrm{E}+06$ & 640 & 0.4 \\
23.710 & $0.837 \mathrm{E}+05$ & 640 & 0.7 \\
23.867 & $0.450 \mathrm{E}+05$ & 480 & 0.2 \\
\hline
\end{tabular}

TABLE $7 \mathrm{~m} / \mathrm{a}=15$ (Quality $=5$ )

\begin{tabular}{|c|c|c|c|}
\hline Decimal Hrs & Distance (km) & $\begin{array}{c}\text { Total } \\
\text { Time }(\mathrm{s})\end{array}$ & $\begin{array}{c}\text { Density } \\
\left(\mathrm{cm}^{-3}\right)\end{array}$ \\
\hline 23.534 & $0.127 \mathrm{E}+06$ & 640 & 0.4 \\
23.710 & $0.837 \mathrm{E}+05$ & 640 & 1.1 \\
23.867 & $0.450 \mathrm{E}+05$ & 480 & 0.8 \\
\hline
\end{tabular}


TABLE 8. $\mathrm{m} / \mathrm{q}=16$ (Quality $=2$ )

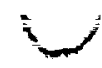

\begin{tabular}{|c|c|c|c|}
\hline Decimal Hrs & Distance $(\mathrm{km})$ & $\begin{array}{c}\text { Total } \\
\text { Time }(\mathrm{s})\end{array}$ & $\begin{array}{c}\text { Density } \\
\left(\mathrm{cm}^{-3}\right)\end{array}$ \\
\hline 23.087 & $0.237 \mathrm{E}+06$ & 512 & 3.8 \\
23.227 & $0.203 \mathrm{E}+06$ & 512 & 6.3 \\
23.372 & $0.167 \mathrm{E}+06$ & 512 & 13.0 \\
23.463 & $0.144 \mathrm{E}+06$ & 128 & 16.7 \\
23.499 & $0.136 \mathrm{E}+06$ & 128 & 20.4 \\
23.534 & $0.127 \mathrm{E}+06$ & 128 & 21.2 \\
23.570 & $0.118 \mathrm{E}+06$ & 128 & 17.7 \\
23.605 & $0.110 \mathrm{E}+06$ & 128 & 17.4 \\
23.641 & $0.101 \mathrm{E}+06$ & 128 & 23.5 \\
23.676 & $0.921 \mathrm{E}+05$ & 128 & 31.8 \\
23.712 & $0.832 \mathrm{E}+05$ & 128 & 28.4 \\
\hline 23.747 & $0.746 \mathrm{E}+05$ & 128 & 29.8 \\
23.782 & $0.660 \mathrm{E}+05$ & 128 & 23.8 \\
23.824 & $0.556 \mathrm{E}+05$ & 160 & 8.7 \\
23.868 & $0.448 \mathrm{E}+05$ & 160 & 9.5 \\
\hline
\end{tabular}

TABLE $9 \mathrm{~m} / \mathrm{q}=17$ (Qualitv $=2$ )

\begin{tabular}{|c|c|c|c|}
\hline Decimal Hrs & Distance (km) & $\begin{array}{c}\text { Total } \\
\text { Time }(\mathrm{s})\end{array}$ & $\begin{array}{c}\text { Density } \\
\left(\mathrm{cm}^{-3}\right)\end{array}$ \\
\hline 23.087 & $0.237 \mathrm{E}+06$ & 512 & 2.2 \\
23.227 & $0.203 \mathrm{E}+06$ & 512 & 3.9 \\
23.372 & $0.167 \mathrm{E}+06$ & 512 & 8.7 \\
23.463 & $0.144 \mathrm{E}+06$ & 128 & 15.2 \\
23.499 & $0.136 \mathrm{E}+06$ & 128 & 14.0 \\
23.534 & $0.127 \mathrm{E}+06$ & 128 & 17.4 \\
23.570 & $0.118 \mathrm{E}+06$ & 128 & 21.2 \\
23.605 & $0.110 \mathrm{E}+06$ & 128 & 18.7 \\
23.641 & $0.101 \mathrm{E}+06$ & 128 & 27.6 \\
23.676 & $0.921 \mathrm{E}+05$ & 128 & 27.5 \\
23.712 & $0.832 \mathrm{E}+05$ & 128 & 31.8 \\
23.747 & $0.746 \mathrm{E}+05$ & 128 & 29.9 \\
23.782 & $0.660 \mathrm{E}+05$ & 128 & 26.8 \\
23.824 & $0.556 \mathrm{E}+05$ & 160 & 12.6 \\
23.868 & $0.448 \mathrm{E}+05$ & 160 & 13.7 \\
\hline
\end{tabular}


TABLE 10. $\mathrm{m} / \mathrm{a}=18$ (Quality $=2$ )

\begin{tabular}{|c|c|c|c|}
\hline Decimal Hrs & Distance $(\mathrm{km})$ & $\begin{array}{c}\text { Total } \\
\text { Time }(\mathrm{s})\end{array}$ & $\begin{array}{c}\text { Density } \\
(\mathrm{cm}-3)\end{array}$ \\
\hline 23.087 & $0.237 \mathrm{E}+06$ & 512 & 0.6 \\
23.227 & $0.203 \mathrm{E}+06$ & 512 & 1.6 \\
23.372 & $0.167 \mathrm{E}+06$ & 512 & 5.7 \\
23.463 & $0.144 \mathrm{E}+06$ & 128 & 12.7 \\
23.499 & $0.136 \mathrm{E}+06$ & 128 & 11.8 \\
23.534 & $0.127 \mathrm{E}+06$ & 128 & 13.8 \\
23.570 & $0.118 \mathrm{E}+06$ & 128 & 15.1 \\
23.605 & $0.110 \mathrm{E}+06$ & 128 & 20.2 \\
23.641 & $0.101 \mathrm{E}+06$ & 128 & 28.6 \\
23.676 & $0.921 \mathrm{E}+05$ & 128 & 27.6 \\
23.712 & $0.832 \mathrm{E}+05$ & 128 & 33.9 \\
23.747 & $0.746 \mathrm{E}+05$ & 128 & 38.3 \\
23.782 & $0.660 \mathrm{E}+05$ & 128 & 43.9 \\
23.824 & $0.556 \mathrm{E}+05$ & 160 & 17.2 \\
23.868 & $0.448 \mathrm{E}+05$ & 160 & 32.1 \\
\hline
\end{tabular}

TABLE 11. $\mathrm{m} / \mathrm{q}=28$ (Quality $=4$ )

\begin{tabular}{|c|c|c|c|}
\hline Decimal Hrs & Distance $(\mathrm{km})$ & $\begin{array}{c}\text { Total } \\
\text { Time }(\mathrm{s})\end{array}$ & $\begin{array}{c}\text { Density } \\
\left(\mathrm{cm}^{-3}\right)\end{array}$ \\
\hline 23.302 & $0.184 \mathrm{E}+06$ & 1016 & 2.6 \\
23.499 & $0.136 \mathrm{E}+06$ & 380 & 7.4 \\
23.677 & $0.918 \mathrm{E}+05$ & 380 & 20.5 \\
23.810 & $0.591 \mathrm{E}+05$ & 396 & 12.1 \\
\hline
\end{tabular}

TABLE 12. $\mathrm{m} / \mathrm{q}=29$ (Quality $=5$ )

\begin{tabular}{|c|c|c|c|}
\hline Decimal Hrs & Distance $(\mathrm{km})$ & $\begin{array}{c}\text { Total } \\
\text { Time }(\mathrm{s})\end{array}$ & $\begin{array}{c}\text { Density } \\
\left(\mathrm{cm}^{-3}\right)\end{array}$ \\
\hline 23.302 & $0.184 \mathrm{E}+06$ & 1016 & 0.8 \\
23.499 & $0.136 \mathrm{E}+06$ & 380 & 3.7 \\
23.677 & $0.918 \mathrm{E}+05$ & 380 & 5.9 \\
23.810 & $0.591 \mathrm{E}+05$ & 396 & 2.7 \\
\hline
\end{tabular}

TABLE 13. $\mathrm{m} / \mathrm{q}=32$ (Quality $=3$ )

\begin{tabular}{|c|c|c|c|}
\hline Decimal Hrs & Distance (km) & $\begin{array}{c}\text { Total } \\
\text { Time (s) }\end{array}$ & $\begin{array}{c}\text { Density } \\
\left(\mathrm{cm}^{-3}\right)\end{array}$ \\
\hline 23.302 & $0.184 \mathrm{E}+06$ & 1016 & 2.0 \\
23.499 & $0.136 \mathrm{E}+06$ & 380 & 4.7 \\
23.677 & $0.918 \mathrm{E}+05$ & 380 & 8.8 \\
23.810 & $0.591 \mathrm{E}+05$ & 396 & 6.7 \\
\hline
\end{tabular}




\section{REFERENCES}

Alfvén, H. 1954, On the Origin of the Solar System, Oxford Univ. Press.

Allen, M., et al. 1987, Astr. Ap., 187, 502.

Balsiger, H. 1990, in Comet Halley 1986, World-Wide Investigation, Results, and Interpretations (Chichester, UK: Ellis Horwood Limited), in press

Balsiger, H., et al. 1986, Nature, 321, 330.

Balsiger, H., et al. 1987a, Astr. Ap., 187, 163.

Balsiger, H., et al. 1987b, J. Phys. E, 20, 759.

d'Uston, C., et al. 1987, Astr. Ap., 187, 137.

Eberhardt, P., et al. 1987, Astr. Ap., 187, 435.

Eviatar, A., et al. 1989, Ap. J., 339, 545.

Feldman, P. D., et al. 1987, Astr. Ap., 187, 325.

Formisano, V., Galeev, A. A., and Sagdeev, R. Z. 1982, Planet. Space Sci., 30, 491.

Fuselier, S. A., et al. 1988, Geophys. Res. Lett., 15, 549.

Fuselier, S. A., et al. 1990, in preparation.

Geiss, J. 1987, Astr. Ap., 187, 859.

Galeev, A. A., et al. 1986, Geophys. Res. Lett., 13, 845.

Gringauz, K. I., et al. 1986, Geophys. Res. Lett., 13, 613.

Huebner, W. F., et al. 1989, Adv. Space Res., 9, (3)385.

Ip, W.-H. 1979, Planet. Space Sci., 27, 121.

Ip, W.-H. 1989a, Adv. Space Res., 9, (3)141.

Ip, W.-H. 1989b, Ap. J., 343, 946.

Ip, W.-H. 1990, Ap. J., 353, 290.

Kettmann, G., et al. 1990, Ann. Geophys, 8, 229.

Kirsch, E., et al. 1989, Ann. Geophys., 7, 107.

Kissel, J., et al. 1986, Nature, 321, 280. 
Korth, A., et al. 1987, Astr. Ap., 187, 149.

Krankowsky, D., et al. 1986, Nature, 321, 326.

Lämmerzahl, P., et al. 1987, Astr. Ap., 187, 169.

Marconi, M. L., and Mendis, D. A. 1988, Ap. J., 330, 513.

Millis, R. L., and Schleicher, D. G. 1986, Nature, 324, 646.

Neubauer, F. M., et al. 1986, Nature, 321, 352.

Neubauer, F. M. 1987, Astr. Ap., 187, 73.

Neubauer, F. M. 1988, J. Geophys. Res., 93, 7272.

Neugebauer, M., et al. 1989, J. Geophys. Res., 94, 1261.

Ogilvie, K. W., Coplan, M. A., Bochsler, P., and Geiss, J. 1986, Science, 232, 374.

Raeder, J., et al. 1989, Chapman Conf. Cometary Plasma Processes, in press.

Reme, H., et al. 1987, Astr. Ap., 187, 33.

Schmidt, H. U., Wegmann, R., Huebner, W. F., and Boice, D. C. 1988, Computer Phys. Comm., 49, 17.

Schwenn, R., et al. 1987, Astr. Ap, 187, 160.

Shelley, E. G., et al. 1987, Astr. Ap., 187, 304

Verigin, M. I., Axford, W. I., Gringauz, K. I., and Richter, A. K. 1987, Geophys. Res. Lett., 14, 987.

Wyckoff, S., and Lindholm, E. 1989, Adv. Space Res., 9, (3)151. 


\section{FIGURE CAPTIONS}

Fig. 1. Total counts, after correction for background, versus mass-anode number for the time (distance) interval $2301-2353$ UT $(254,000-39,600 \mathrm{~km})$. Only those counts in the two elevation-angle bins closest to the spacecraft ram direction are included. The heavy and medium mode data are plotted separately. The numbers over the count-rate spectra indicate the mass/charge values of each of the peaks. The background correction averaged about 30 counts/anode.

Fig. 2. Ilustration of a least-squares fit of the countrate vs mass-anode spectrum for $\mathrm{m} / \mathrm{q}=$ 16,17 , and 18 amu/e. The observed counts are indicated by circles; the solid curve is the least-squares fit; and the dotted curves indicate the contributions of the individual m/q peaks to the fit. The fitting procedure solved for 3 parameters (the heights of the peaks with $\mathrm{m} / \mathrm{q}$ $=16,17$, and $18 \mathrm{amu} / \mathrm{e}$, respectively) which gave the best fit to the 10 measured points. The time (distance) interval for the data displayed here was 23.372 UT $(167,000 \mathrm{~km})$.

Fig 3. Observed densities of protons (circles) and ions with mass/charge $=2 \mathrm{amu} / \mathrm{e}$ (triangles) versus distance. The continuous curve indicates the proton density from the model of Schmidt et al. (1988). The vertical dashed line marks the location of the magnetic pileup boundary.

Fig. 4. Density versus log distance profiles for ions with 12 different values of mass/charge. The circles represent the hot-ion densities observed by HERS. The continuous curves show the densities from the model of Schmidt et al. (1988). The vertical dashed line marks the location of the magnetic pileup boundary. 
Fig. 5. Ratios of the densities of ions with different values of mass/charge versus log cometocentric distance. The circles represent the hot-ion densities observed by HERS. The condinuous curves show the densities from the model of Schmidt et al. (1988). The vertical dashed line marks the location of the magnetic pileup boundary. 
B. E. GOLDSTEIN, R. GOLDSTEIN, and M. NEUGEBAUER: Mail Stop 169-506, Jet Propulsion Laboratory, 4800 Oak Grove Drive, Pasadena, CA 91109

S. A. FUSELIER: Dept. 91-20, BIdg. 255, Lockheed Palo Alto Research Laboratory, 3251 Hanover St., Palo Alto, CA 94304

H. BALSIGER: Physikalisches Institut, University of Bem, Sidlerstrasse 5, CH-3012 Bern, Switzerland

W.-H. IP: Max-Planck-Institut für Aeronomie, D-3411 Katlenburg-Lindau, Federal Republic of Germany 

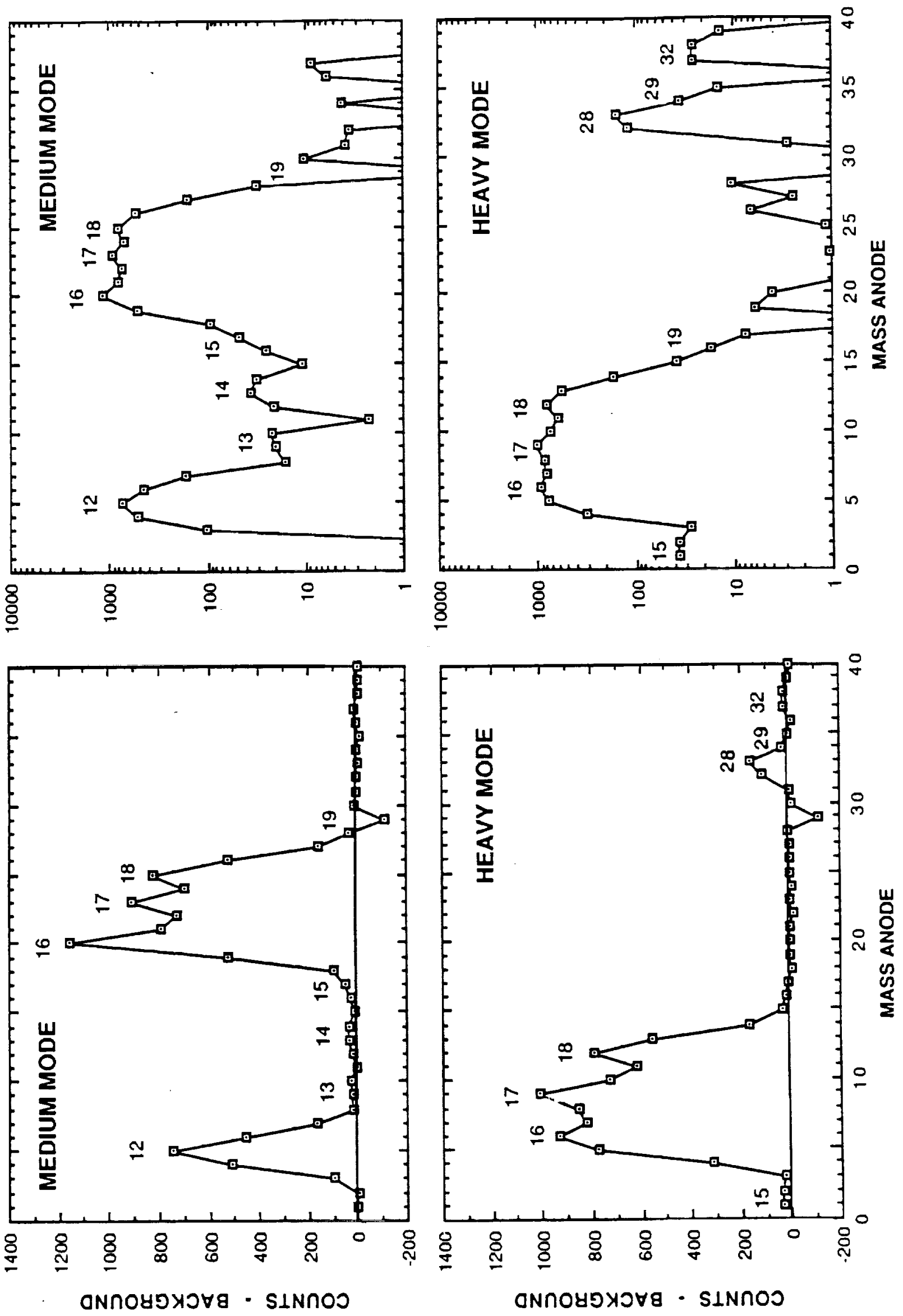


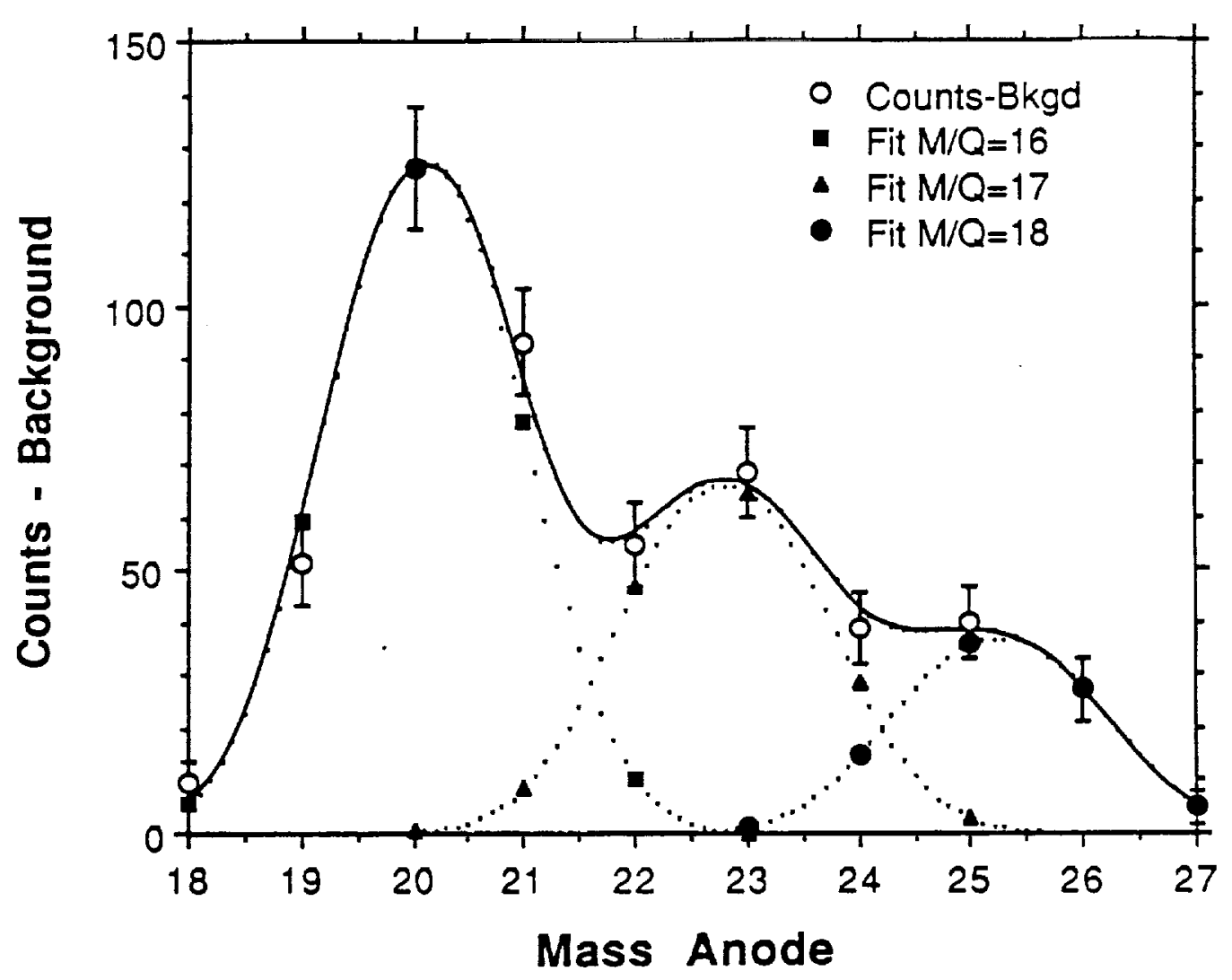

Figure 2 


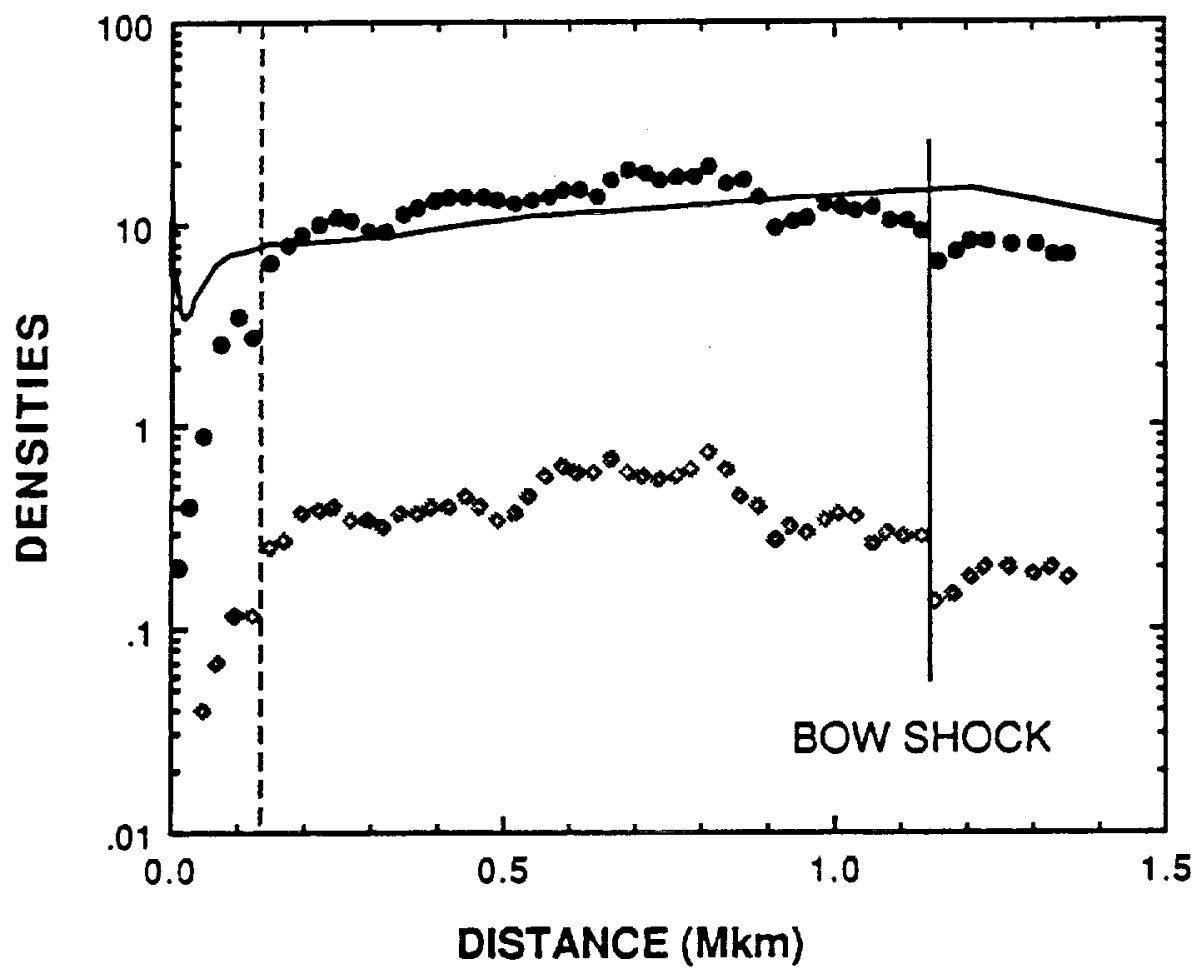

Figure 3 

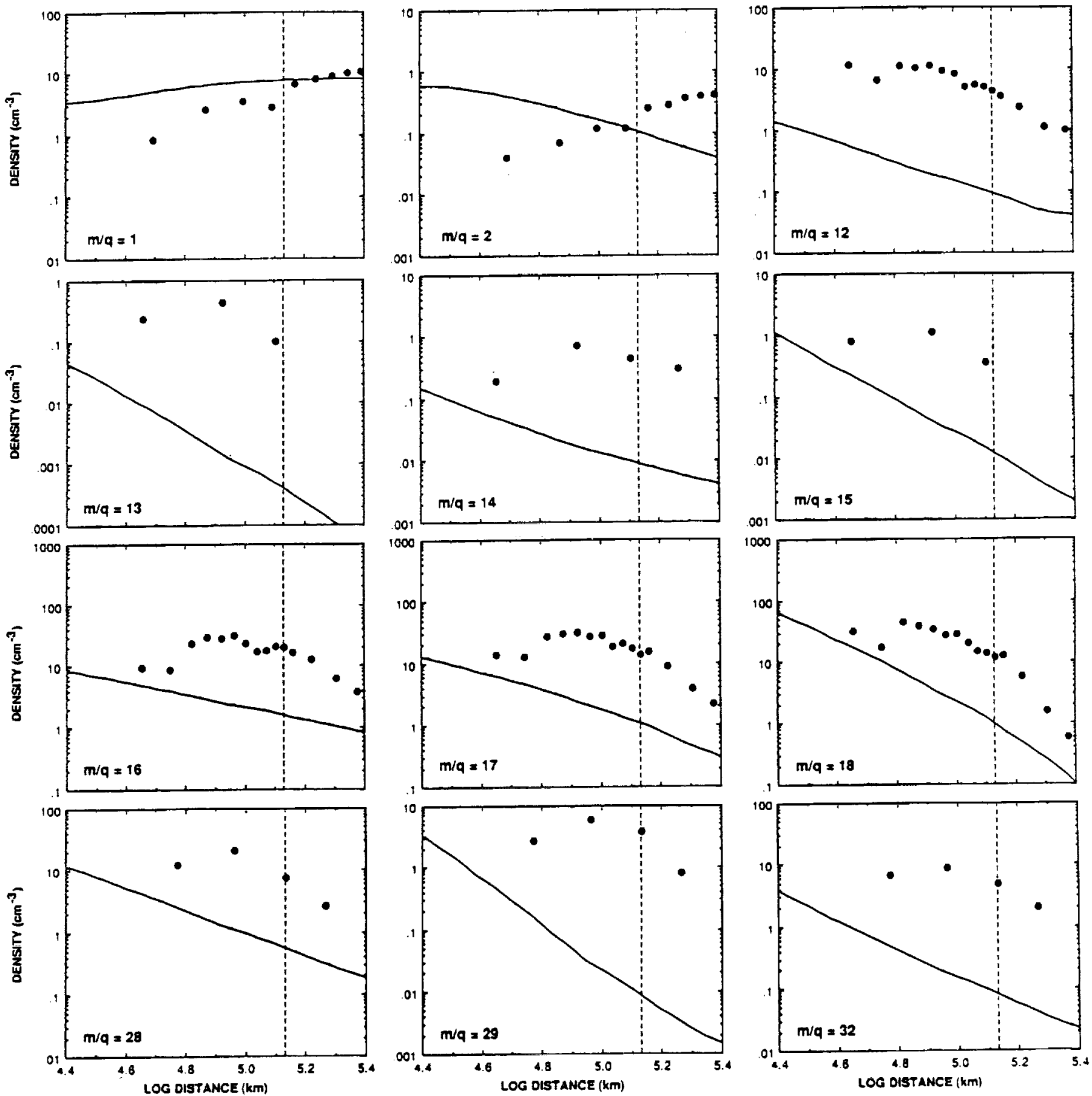

Figure 4 

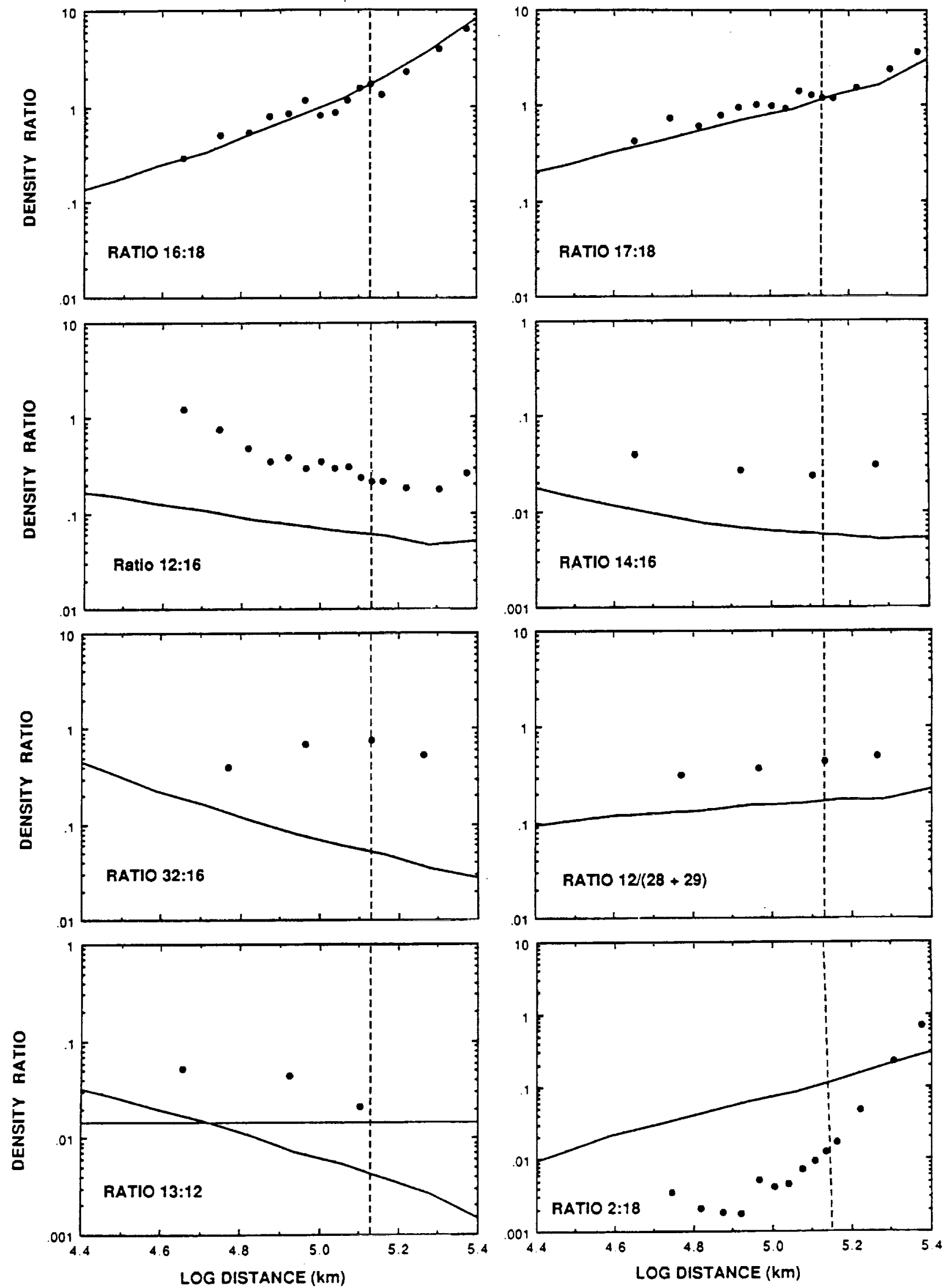

Figure 5 